\title{
ẢNH HƯỞNG CỦA NHẬN THỨC TRÁCH NHIÊMM XÃ HỘI DOANH NGHIỆP ĐẾN NIỀM TIN VÀO TỔ CHỨC CỦA NHÂN VIÊN NGÂN HÀNG THƯƠNG MẠI CỔ PHẦN TẠI THÀNH PHỐ SÓC TRĂNG \\ Hà Nam Khánh Giao ${ }^{1}$ - Trịnh Thị Hồng Tiên²
}

\section{TÓM TẮT}

Nghiên cứu kiểm định mức độ tác động của các nhân tố trách nhiệm xã hội doanh nghiẹp (Corporate Social Responsibility-CSR) đến niềm tin của nhân viên các ngân hàng thương mai cổ phần (TMCP) vào tổ chức tại thành phố Sóc Trăng, bằng việc khảo sát 210 nhân viên. Phuoong pháp kiểm định thang đo bằng hệ số Cronbach's Alpha, phân tích EFA cùng với phân tích hồi quy bội được sử dụng.

Kết quả cho thấy mức độ ảnh hưởng của 04 nhân tố CSR đến niềm tin của nhân viên các ngân hàng TMCP vào tổ chức tại thành phố Sóc Trăng - theo tầm quan trọng giảm dần: Trách nhiệm pháp luật, Trách nhiệm đạo đức, Trách nhiệm thiện nguyện, Trách nhiệm kinh tế. Nghiên cứu đề ra một số hàm ý quản trị cho các nhà quản lý ngân hàng nhằm tăng cuờng niềm tin của nhân viên.

Tù̀ khóa: Trách nhiệm xã hội doanh nghiệp, Trách nhiệm pháp luật, Trách nhiệm đạo đức, Trách nhiệm thiện nguyện, Trách nhiệm kinh tế

\section{THE AFFECT OF CORPORATE SOCIAL RESPONSIBILITY ON STAFF BELIEF TO THE COMMERCIAL BANKS IN SOC TRANG CITY ABSTRACT}

The research investigates how the factors of Corporate Social Responsibility (CSR) affect staff belief to the commercial banks in Sóc Trăng province, by intervewing 210 staff. The method of Cronbach's Alpha, EFA analysis and multiple regression analysis were used.

The result shows that the affects of 04 factors on staff belief to the commercial banks in Sóc Trăng province decreasingly: Legal responsibility, Ethical responsibility, Philanthropic responsibility, Economic responsibility. The research also suggests some solutions to the bank management to enhance staff belief.

Keywords: Corporate Social Responsibility, Legal responsibility, Ethical responsibility, Philanthropic responsibility, Economic responsibility

\section{GIỚI THIÊUU}

Vấn đề "trách nhiệm xã hội doanh nghiệp" (Corporate Social Responsibility CSR) được Bowen (1953) đưa ra, và trở thành một chủ đề nóng của các nhà kinh doanh, các nhà nghiên cứu, của cộng đồng và của xã hội. Tại Việt Nam $(\mathrm{VN}), \mathrm{CSR}$ chỉ phổ biến ở các năm gần đây và hầu như chỉ có các doanh nghiệp lớn quan tâm, xem

\footnotetext{
$\checkmark \quad{ }^{1}$ Trưởng khoa Đào tạo Sau đại học - Trường Đại học Tài Chính - Marketing

$\checkmark$ E-mail: khanhgiaohn@yahoo.com

$\checkmark$ Điện thoại di động: 0903306363

$\checkmark$ Địa chỉ liên lạc thư từ: A65 khu phố Nam Thông 1, Phú Mỹ Hưng, P. Tân Phú, Q.7, TPHCM.

$\checkmark \quad{ }^{2}$ Nơi công tác: Ngân hàng Hợp Tác Xã Việt Nam chi nhánh Sóc Trăng

$\checkmark \quad$ E-mail: tientth1984@gmail.com

$\checkmark$ Điện thoại di động: 0949610007

$\checkmark$ Địa chỉ liên lạc thư từ: số 32 đường Nguyễn Hùng Phước, P. 1, TP. Sóc Trăng.
} 
như là một trong những triết lý kinh doanh cơ bản và luôn đi cùng với chiến lược phát triển, góp phần quan trọng vào sự thành công vững chắc, giúp doanh nghiệp thực hiện được tầm nhìn, sứ mệnh của mình.

Mặc dù nhận thức về vai trò của CSR tại Việt Nam trong thời gian gần đây tương đối được các doanh nghiệp quan tâm và tìm hiểu, nhưng số lượng các nghiên cứu sâu về CSR vẫn chưa được thực hiện nhiều, đa số nhằm tổng kết những hoạt động của các tổ chức quốc tế và trong nước trong việc thúc đẩy việc thực hiện CSR tại Việt Nam, và đề cập đến những khuôn khổ pháp lý về CSR làm căn cứ triển khai các chương trình CSR tại các doanh nghiệp. Hoàng Thị Phương Thảo và Huỳnh Long Hồ (2015) là nghiên cứu hầu như duy nhất tiếp cận theo góc nhìn của nhân viên trong tổ chức kinh doanh.

Ngân hàng là một lĩnh vực kinh doanh điển hình, mà trong đó vai trò CSR càng được nhấn mạnh. Các ngân hàng xem CSR là một chiến lược dài hạn giúp tạo ra giá trị của doanh nghiệp, đồng thời xây dựng được lòng tin và sự tôn trọng của người tiêu dùng, đối tác nói riêng và cộng đồng xã hội nói chung. Bài viết chọn nghiên cứu tác động của nhận thức CSR đến niềm tin vào tổ chức của nhân viên Ngân hàng TMCP tại TP. Sóc Trăng nhằm bổ sung, cung cấp cho giới học thuật và các doanh nghiệp những kết quả thực nghiệm về mối quan hệ này. Đây chính là "chìa khóa" quan trọng để giúp các ngân hàng có thể đạt được các mục tiêu quan trọng như: tăng năng suất, hiệu quả công việc, tăng trưởng doanh thu, duy trì và gia tăng lợi nhuận...

\section{TỔNG QUAN LÝ LUẬN}

\subsection{Trách nhiệm xã hội của doanh nghiệp (CSR)}

Bowen (1953) cho rằng "CSR đề cập đến nghĩa vụ của doanh nghiệp để theo đuổi những chính sách, để thực hiện những quyết định, hoặc thực hiện những hoạt động để đạt được các mục tiêu đặt ra và những giá trị xã hội của chúng tôi". Carroll (1979) cho rằng CSR bao gồm sự mong đợi của xã hội về kinh tế, pháp luật, đạo đức và lòng từ thiện đối với các tổ chức tại một thời điểm nhất định, và Caroll (1991) đưa ra mô hình kim tự tháp về CSR (Hình 1) được nhiều người đồng tình, theo đó, CSR bao gồm các thành phần: (1) Trách nhiệm kinh tế (economic responsibility): tối đa hóa lợi nhuận, cạnh tranh, hiệu quả và tăng trưởng, (2) Trách nhiệm pháp luật/pháp lý (legal responsibility): nghĩa vụ tuân theo các luật lệ, luật pháp của địa phương, của đất nước và cả luật quốc tế, (3) Trách nhiệm đạo đức (ethical responsibility): những quy tắc, giá trị được xã hội chấp nhận nhưng chưa được đưa vào văn bản luật, (4) Trách nhiệm thiện nguyện/từ thiện (philanthropic responsibility): những hành vi của doanh nghiệp vượt ra ngoài sự trông đợi của xã hội, như quyên góp ủng hộ người khó khăn, tài trợ học bổng, đóng góp cho các dự án cộng đồng...

\section{Trách nhiệm thiện nguyện/từ thiện}

Là một công dân doanh nghiệp tốt

Có đóng góp nguồn lực cho cộng đồng, cải thiện chất lượng cuộc sống

\section{Trách nhiệm đạo đức}

Thực hiện đúng những gì được coi là công bằng, đúng đắn, hợp lý

Trách nhiệm pháp luật/pháp lý

Cần phải hoạt động theo đúng pháp luật 


\section{Hình 1: Mô hình kim tự tháp về CSR}

Nguồn: Carroll (1991)

Lợi ích của việc thực hiện CSR: (1) Giảm chi phí và tăng năng suất, (2) Tăng doanh thu, (3) Nâng cao giá trị thương hiệu và uy tín của công ty, (4) Thu hút nguồn lao động giỏi, (5) Cơ hội tiếp cận thị trường mới, (6) Khai thác các cơ hội từ việc thực hiện trách nhiệm xã hội, (7) Sự trung thành của nhân viên và khách hàng (Nguyễn Đình Cung và Lưu Đức Minh, 2008).

\subsection{Tác động của CSR đến niềm tin vào tổ chức}

Có rất nhiều công trình nghiên cứu của các tác giả khác nhau về niềm tin tổ chức. Trong hành vi tổ chức, niềm tin được mô tả là một khái niệm phức tạp được cho là trung tâm của mối quan hệ giữa các cá nhân mà đó là đặc trưng của tổ chức (Tschannen-Moran \& ctg, 1998).

Niềm tin tổ chức là một khái niệm chứa đựng nhiều nội dung. Theo cách nghĩ thông thường, niềm tin thường gắn với mối quan hệ con người và mang ý nghĩa một chiều. Tuy nhiên, xét phạm vi một tổ chức, niềm tin tổ chức được nhận thức với ý nghĩa rộng hơn: (i) niềm tin tổ chức là hệ quả của mối quan hệ con người trong tổ chức ở cùng một cấp (đồng nghiệp) và giữa nhiều cấp độ khác nhau (nhóm, trên-dưới, đơn vị, tổ chức, đối tượng hữu quan bên ngoài); (ii) Niềm tin tổ chức được hình thành trên cơ sở những giá trị văn hóa tổ chức (văn hóa doanh nghiệp) đặc trưng như chuẩn mực hành vi, giá trị cốt lõi, niềm tin; (iii) Niềm tin là hệ quả của mối quan hệ giao tiếp giữa các cá nhân, ví dụ như cung cấp thông tin chính xác, tính tường minh trong các quyết định, thể hiện sự chân thành và cởi mở một cách phù hợp; (iv) Niềm tin được tạo dựng thông qua một quá trình xây dựng, ổn định và điều chỉnh; vì thế, lòng tin không bất biến; (v) Niềm tin của một cá nhân chịu tác động bởi nhiều yếu tố, từ nhận thức, cảm xúc đến hành động, ở các cấp độ khác nhau (Nguyễn Mạnh Quân, 2013).

Mặc dù cách diễn đạt là khác nhau, các định nghĩa về lòng tin tổ chức vẫn thể hiện sự thống nhất về những khái niệm bản chất của lòng tin tổ chức như niềm tin (belief), sự tự nguyện (willingness), chấp nhận thiệt thòi, rủi ro (vulnerability). Niềm tin tổ chức là sự tự nguyện của các thành viên trong tổ chức chấp nhận chịu thiệt thòi một cách hợp lý trong việc tin tưởng rằng cá nhân, nhóm cá nhân hay tổ chức khác có khả năng hành động một cách hiệu quả, ứng xử cởi mở và trung thực, không vị kỷ (quan tâm đến những người khác), đáng tin cậy và có bản sắc riêng trong việc thể hiện các chuẩn mực và giá trị chung, và trong việc phấn đấu các mục tiêu chung (Mishra, 1996 trích bởi Hoàng Thị Phương Thảo và Huỳnh Long Hồ, 2015).

Nghiên cứu của Lee \& ctg (2012) cho thấy bốn thành phần của CSR (kinh tế, pháp lý, đạo đức và từ thiện) có tác dụng khác nhau về chất lượng mối quan hệ của các nhân viên của các doanh nghiệp dịch vụ thực phẩm nhượng quyền thương mại đặt tại Hàn Quốc. Nghiên cứu của Mirza \& Redzuan (2012) cho thây có một mối quan hệ tích 
cực cao giữa niềm tin và cam kết của giáo viên với tổ chức trong các trường tiểu học ở tỉnh Golestan, Iran. Nghiên cứu của You \& ctg (2013) cho thấy CSR đã có một tác động đáng kể vào sự hài lòng công việc và cam kết tổ chức của nhân viên bộ phận kinh doanh của một công ty bảo hiểm.

Ở Việt Nam, nghiên cứu của Hoàng Thị Phương Thảo \& Huỳnh Long Hồ (2015) cho thấy CSR có tác động tích cực đến niềm tin của nhân viên 05 ngân hàng TMCP tại TPHCM, từ đó, có tác động tích cực đến cam kết gắn bó của nhân viên.

\subsection{Mô hình nghiên cứu đề xuất}

Mô hình được đề nghị nghiên cứu trong bài viết này được chọn từ mô hình trách nhiệm xã hội doanh nghiệp của Caroll (1979) với 4 thành phần tác động, được mô tả trong Hình 2.

Giả thuyết $H_{1}(+)$ : Nhận thức trách nhiệm kinh tế có ảnh huoởng tích cực đến niềm tin vào tổ chức của nhân viên với ngân hàng

Giả thuyết $H_{2}(+)$ : Nhận thức trách nhiệm pháp luật có ảnh hưởng tích cực đến niềm tin vào tổ chức của nhân viên với ngân hàng

Giả thuyết $H_{3}(+)$ : Nhận thức trách nhiệm đạo đức có ảnh hưởng tích cực đến niềm tin vào tồ chức của nhân viên với ngân hàng

Giả thuyết $H_{4}(+)$ : Nhận thí̛c trách nhiệm thiện nguyện có ảnh hưởng tích cực đến niềm tin vào tổ chức của nhân viên với ngân hàng

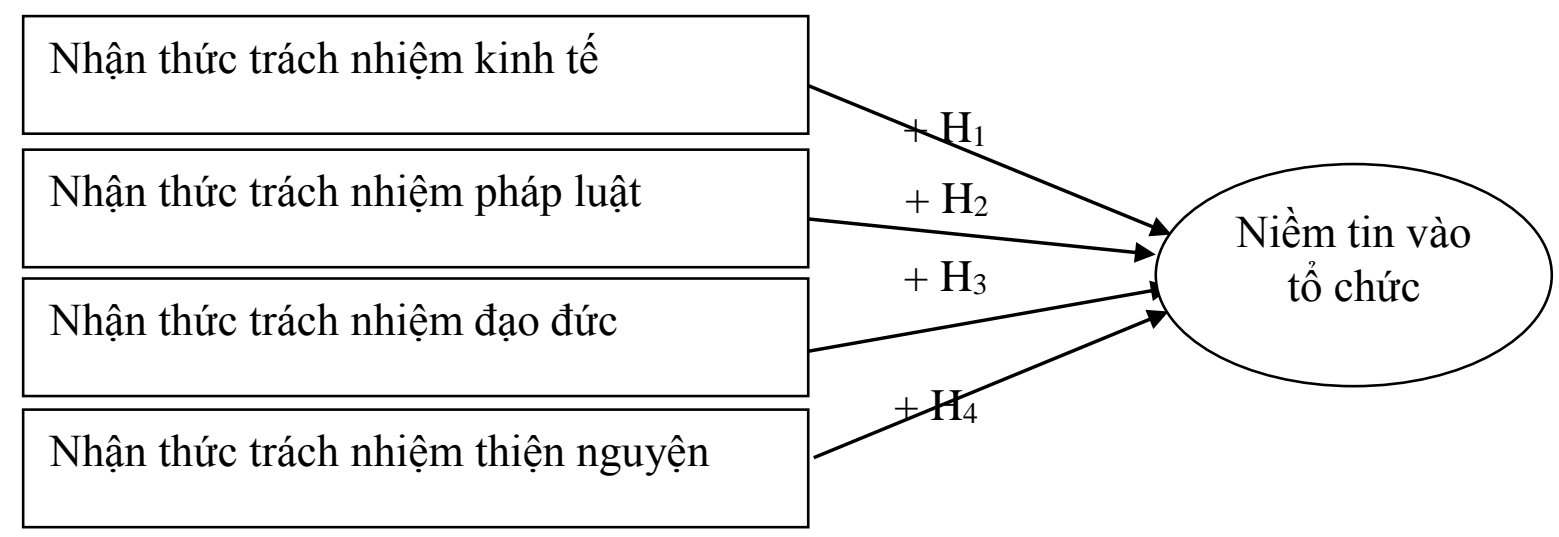

\section{Hình 2: Mô hình nghiên cứu đề xuất}

(Nguồn: nhóm tác giả đề xuất)

\section{KẾT QUẢ NGHIÊN CÚU}

\subsection{Thống kê mẫu nghiên cứu}

Đối tượng khảo sát là các nhân viên đang làm việc tại các chi nhánh và phòng giao dịch của 04 ngân hàng $\mathrm{TMCP}$ trên địa bàn TP. Sóc Trăng: ngân hàng Vietcombank đại diện nhóm 1 (vốn điều lệ lớn hơn 20 nghìn tỷ đồng), ngân hàng Techcombank đại diện nhóm 2 (vốn điều lệ từ 5 nghìn tỷ đển dưới 20 nghìn tỷ đồng), ngân hàng $\mathrm{AB}$ Bank và ngân hàng Phương Đông đại diện nhóm 3 (vốn điều lệ từ 3,5 nghìn tỷ đến dưới 5 nghìn tỷ đồng), không chọn khảo sát ngân hàng đại diện nhóm 4 (vốn điều lệ dưới 3,5 nghìn tỷ đồng) vì vấn đề CSR và niềm tin chưa được đạt mức quan tâm cao của các ngân hàng trong nhóm.

Mẫu được chọn theo phương pháp thuận tiện. Khảo sát được thực hiện bằng cách gửi bảng câu hỏi trực tiếp, gửi thư điện tử và khảo sát qua công cụ Google.docs đến nhân viên tại các ngân hàng. Với 31 biển quan sát ban đầu, số quan sát cần thiết là 
trên 155 (Hoàng Trọng và Chu Nguyễn Mộng Ngọc, 2008), tổng số bảng câu hỏi gởi đi là 250 , thu về 225 (tỷ lệ $90 \%$ ), sau khi sàng lọc, còn 210 bảng khảo sát hợp lệ được sử dụng cho nghiên cứu. Thống kê mô tả mẫu như trong Bảng 1 .

Bảng 1: Bảng thống kê mô tả khảo sát

\begin{tabular}{|c|c|c|c|}
\hline \multicolumn{2}{|c|}{ Mẫu n = 210} & Tần số & $\%$ \\
\hline \multirow[t]{2}{*}{ Giới tính } & Nam & 104 & 49,5 \\
\hline & Nũ & 106 & 50,5 \\
\hline \multirow{4}{*}{ Ngân hàng } & Vietcombank & 93 & 44,3 \\
\hline & Techcombank & 34 & 16,2 \\
\hline & $\mathrm{AB}$ bank & 55 & 26,2 \\
\hline & OCB & 28 & 13,3 \\
\hline \multirow{3}{*}{ Tuổi } & Từ 18 đến 30 tuổi & 84 & 40,0 \\
\hline & Từ 31 đến 40 tuổi & 73 & 34,8 \\
\hline & Từ 41 đến 50 tuổi & 53 & 25,2 \\
\hline \multirow{3}{*}{ Trình độ học vấn } & Trung cấp - cao đẳng & 65 & 31,0 \\
\hline & Đại học & 126 & 60,0 \\
\hline & Trên đại học & 19 & 9,0 \\
\hline \multirow{5}{*}{ Kinh nghiệm } & Dưới 1 năm & 9 & 4,3 \\
\hline & Từ 1 đến dưới 3 năm & 59 & 28,1 \\
\hline & Từ 3 đến 5 năm & 72 & 34,3 \\
\hline & Từ 5 đến dưới 15 năm & 62 & 29,5 \\
\hline & Trên 15 năm & 8 & 3,8 \\
\hline \multirow{10}{*}{ Vị trí làm việc } & Nhân sự hành chính & 9 & 4,3 \\
\hline & Tín dụng & 20 & 9,5 \\
\hline & Giao dịch viên & 28 & 13,3 \\
\hline & Thanh toán quốc tế & 27 & 12,9 \\
\hline & Phát triển sản phẩm & 12 & 5,7 \\
\hline & Quan hệ khách hàng & 39 & 18,6 \\
\hline & Kiểm soát viên & 22 & 10,5 \\
\hline & Thẩm định tín dụng & 29 & 13,8 \\
\hline & Kế toán & 14 & 6,7 \\
\hline & Khác & 10 & 4,8 \\
\hline
\end{tabular}

\subsection{Kiểm định độ tin cậy của thang đo}

(Nguồn: Số liệu điều tra của nhóm tác giả)

Kết quả đánh giá các thang đo bằng Cronbach's Alpha (bảng 2) cho thấy các thang đo đều đạt độ tin cậy Cronbach's Alpha $>0,6$ và hệ số tương quan biến - tổng $>$ 0,3 , tất cả các biến quan sát của các thang đo đều thỏa mãn điều kiện để phân tích EFA.

Bảng 2: Thống kê kiểm định độ tin cậy Cronbach's Alpha

\begin{tabular}{|l|c|c|c|c|}
\hline \multicolumn{1}{|c|}{ Thang đo } & $\begin{array}{c}\text { Ký } \\
\text { hiệu }\end{array}$ & $\begin{array}{c}\text { Số biến } \\
\text { quan sát }\end{array}$ & $\begin{array}{c}\text { Cronbach' } \\
\text { s Alpha }\end{array}$ & $\begin{array}{c}\text { Hệ số tương quan } \\
\text { biến-tổng nhỏ nhất }\end{array}$ \\
\hline Trách nhiệm kinh tế & KT & 6 & 0,840 & 0,536 \\
\hline Trách nhiệm pháp luật & PL & 4 & 0,861 & 0,623 \\
\hline Trách nhiệm đạo đức & DD & 7 & 0,819 & 0,438 \\
\hline
\end{tabular}




\begin{tabular}{|l|c|c|c|c|}
\hline Trách nhiệm thiện nguyện & $\mathrm{TN}$ & 4 & 0,738 & 0,435 \\
\hline Niềm tin vào tồ chức & $\mathrm{NT}$ & 4 & 0,836 & 0,598 \\
\hline
\end{tabular}

\subsection{Phân tích nhân tố khám phá EFA}

Phương pháp EFA được sử dụng cho 21 biến quan sát của các biến độc lập, sử dụng phương pháp Principal Component Analysis với phép quay Varimax và điểm dừng khi trích các yếu tố có Eigenvalues là 1 . Kết quả phân tích EFA lần 2 với 20 biến quan sát còn lại, hệ số $\mathrm{KMO}=0,835$ đạt yêu cầu $>0,5$ giải thích được kích thước mẫu phù hợp cho phân tích nhân tố và hệ số Barlett có mức ý nghĩa $\mathrm{Sig}=0,000<0,5$ (có sự tương quan giữa các biến) đã khẳng định rằng phương pháp phân tích trên là phù hợp (hay thỏa mãn điều kiện cho phân tích nhân tố). Tổng phương sai trích là $60,253 \%$ tức là 20 biến rút trích ra góp phần giải thích được khoảng $60,253 \%$ sự biến thiên của các biến quan sát và hệ số tải nhân số (factor loading) đều lớn hơn 0,5 nên đạt yêu cầu (Bảng 3).

\section{Bảng 3: Kết quả phép xoay nhân tố lần 2}

\begin{tabular}{|c|c|c|c|c|c|}
\hline \multirow{2}{*}{\multicolumn{2}{|c|}{ Thành phần }} & \multicolumn{4}{|c|}{ Các nhân tố trích } \\
\hline & & 1 & 2 & 3 & 4 \\
\hline Trách nhiệm kinh tế & $\begin{array}{l}\mathrm{KT}_{2} \\
\mathrm{KT}_{4} \\
\mathrm{KT}_{3} \\
\mathrm{KT}_{5} \\
\mathrm{KT}_{1} \\
\mathrm{KT}_{6}\end{array}$ & $\begin{array}{l}0,816 \\
0,813 \\
0,726 \\
0,719 \\
0,669 \\
0,646\end{array}$ & & & \\
\hline Trách nhiệm đạo đức & $\begin{array}{l}\mathrm{DD}_{6} \\
\mathrm{DD}_{2} \\
\mathrm{DD}_{5} \\
\mathrm{DD}_{3} \\
\mathrm{DD}_{7} \\
\mathrm{DD}_{1}\end{array}$ & & $\begin{array}{l}0,753 \\
0,736 \\
0,703 \\
0,692 \\
0,658 \\
0,648\end{array}$ & & \\
\hline Trách nhiệm pháp luật & $\begin{array}{l}\mathrm{PL}_{3} \\
\mathrm{PL}_{5} \\
\mathrm{PL}_{1} \\
\mathrm{PL}_{6}\end{array}$ & & & $\begin{array}{l}0,888 \\
0,812 \\
0,788 \\
0,703\end{array}$ & \\
\hline Trách nhiệm thiện nguyện & $\begin{array}{l}\mathrm{TN}_{5} \\
\mathrm{TN}_{6} \\
\mathrm{TN}_{3} \\
\mathrm{TN}_{4}\end{array}$ & & & & $\begin{array}{l}0,808 \\
0,716 \\
0,589 \\
0,586\end{array}$ \\
\hline Mức ý nghĩa (Sig, trong ki & innh B & & & & 0,000 \\
\hline Hệ số KMO & & & & & 0,835 \\
\hline Tổng phương sai trích & & & & & $60,253 \%$ \\
\hline
\end{tabular}

(Nguồn: Kết quả truy xuất tù̀ SPSS)

Kết quả phân tích nhân tố biến phụ thuộc, ta có hệ số $\mathrm{KMO}=0,692$, và hệ số Barlett có mức ý nghĩa $\mathrm{Sig}=0,000<0,5$, phương sai trích $67,036 \%$ và các biến đều có hệ số tải nhân tố (factor loading) lớn hơn 0,5 , thang đo niềm tin vào tổ chức đạt giá trị 
hội tụ (bảng 4).

Bảng 4: Kết quả phân tích EFA đối với thang đo niềm tin

\begin{tabular}{|l|c|c|}
\hline Thành phần & Các nhân tố trích & Tên nhân tố \\
\cline { 2 - 3 } & 0,877 & \\
$\mathrm{NT}_{2}$ & 0,831 & \multirow{2}{*}{ Niềm tin tổ chức (NT) } \\
$\mathrm{NT}_{5}$ & 0,798 & \\
$\mathrm{NT}_{4}$ & 0,765 & 0,000 \\
$\mathrm{NT}_{3}$ & & 0,692 \\
\hline Mức ý nghĩa (Sig. trong kiểm định Bartlett) & $67,036 \%$ \\
\hline \multicolumn{2}{|l|}{ Hệ số KMO } & \\
\hline \multicolumn{2}{|l|}{ Tồng phương sai trích } \\
\hline
\end{tabular}

\subsection{Phân tích mối tương quan tuyến tính}

(Nguồn: Kết quả truy xuất tù̀ SPSS)

Bảng 5: Ma trận tương quan Pearson

\begin{tabular}{|l|r|r|r|r|r|}
\hline & KT & PL & DD & \multicolumn{1}{c|}{ TN } & NT \\
\hline Kinh tế (KT) & 1 & $0,244^{* *}$ & $0,185^{* *}$ & $0,303^{* *}$ & $0,330^{* *}$ \\
\hline Pháp luật (PL) & & 1 & $0,336^{* *}$ & $0,442^{* *}$ & $0,617^{* *}$ \\
\hline Đạo đức (DD) & & & 1 & $0,502^{* *}$ & $0,519^{* *}$ \\
\hline Thiện nguyện (TN) & & & & & $0,521^{* *}$ \\
\hline Niềm tin (NT) & & & & & 1 \\
\hline
\end{tabular}

**. Tương quan ở mức ý nghĩa 1\% (Kiểm định 2 phía).

(Nguồn: Kết quả truy xuất tù̀ SPSS)

Kết quả phân tích tương quan (bảng 5) chỉ ra rằng các hệ số tương quan đều có ý nghĩa thống kê $(\operatorname{Sig}<0,05)$ nên tất cả các biến sẽ được sử dụng trong phân tích hồi quy ở bước tiếp theo. Tuy nhiên, hệ số tương quan giữa các biến độc lập cao, kiểm định đa cộng tuyến cần được tiến hành trong các bước tiếp theo.

\subsection{Xây dựng mô hình hồi quy tuyến tính}

Bảng 6: Tóm tắt mô hình hồi quy tuyến tính

\begin{tabular}{|c|r|r|r|c|}
\hline Hệ số $\mathbf{R}$ & $\begin{array}{c}\text { Hệ số xác } \\
\text { định }-\mathbf{R}^{\mathbf{2}}\end{array}$ & $\begin{array}{c}\text { Hệ số } \mathbf{R}^{\mathbf{2}} \\
\text { hiệu chỉnh }\end{array}$ & $\begin{array}{c}\text { Sai số chuẩn } \\
\text { của ước lượng }\end{array}$ & $\begin{array}{c}\text { Chỉ số Durbin- } \\
\text { Watson }\end{array}$ \\
\hline $0,727^{\mathrm{a}}$ & 0,528 & 0,519 & 0,40406 & 1,765 \\
\hline
\end{tabular}

Bảng 7: Trọng số hồi quy

(Nguồn: Kết quả truy xuất tù̀ SPSS)

\begin{tabular}{|c|c|c|c|c|c|c|}
\hline \multirow{2}{*}{ Nhân tố } & \multicolumn{2}{|c|}{$\begin{array}{l}\text { Hệ số beta chưa } \\
\text { chuẩn hóa }\end{array}$} & \multirow{2}{*}{$\begin{array}{c}\begin{array}{c}\text { Hệ số beta } \\
\text { chuẩn hóa }\end{array} \\
\text { Beta }\end{array}$} & \multirow{2}{*}{ Giá trị t } & \multirow{2}{*}{$\begin{array}{l}\text { Giá trị } \\
\text { Sig. }\end{array}$} & \multirow{2}{*}{$\begin{array}{c}\text { Hệ số phóng } \\
\text { đại phương } \\
\text { sai - VIF }\end{array}$} \\
\hline & B & $\begin{array}{l}\text { Sai số } \\
\text { chuẩn }\end{array}$ & & & & \\
\hline (Hằng số) & 0,536 & 0,232 & & 2,306 & 0,022 & \\
\hline $\mathrm{KT}^{* *}$ & 0,115 & 0,046 & 0,128 & 2,521 & 0,012 & 1,120 \\
\hline PL*** & 0,429 & 0,055 & 0,424 & 7,788 & 0,000 & 1,290 \\
\hline $\mathrm{DD}^{* * *}$ & 0,176 & 0,036 & 0,274 & 4,887 & 0,000 & 1,366 \\
\hline $\mathrm{TN}^{* * *}$ & 0,150 & 0,057 & 0,157 & 2,621 & 0,009 & 1,567 \\
\hline
\end{tabular}

(Nguồn: Kết quả truy xuất tù̀ SPSS)

Bảng 6 cho thấy hệ số $\mathrm{R}^{2}$ hiệu chỉnh là 0,519 , các biến độc lập giải thích được $51,9 \%$ biến phụ thuộc niềm tin vào tổ chức. Bảng 7 cho thấy các hệ số beta của biến 
độc lập đều có ý nghĩa thống kê (Sig. < 0,05), và hệ số beta dương chứng tỏ có ảnh hưởng thuận chiều đến biến phụ thuộc NT.

Bảng 8: Phân tích ANOVA

\begin{tabular}{|l|r|r|r|r|r|}
\hline & $\begin{array}{c}\text { Tổng bình } \\
\text { phương }\end{array}$ & Bậc tự do & $\begin{array}{c}\text { Trung bình } \\
\text { bình phương }\end{array}$ & Hệ số F & Giá trị Sig. \\
\hline Hồi quy & 37,476 & 4 & 9,369 & 57,386 & $0,000^{\mathrm{b}}$ \\
\hline Phần dư & 33,469 & 205 & 0,163 & & \\
\hline Tổng & 70,945 & 209 & & & \\
\hline
\end{tabular}

(Nguồn: Kết quả truy xuất tùt SPSS)

Bảng 8 cho thấy mô hình hồi quy có kiểm định $\mathrm{F}=57,386$, Sig. $<0,05$ cho thấy mô hình hồi quy tuyến tính bội đã xây dựng phù hợp với tập dữ liệu ở độ tin cậy $95 \%$. Vậy mô hình hồi quy bội chưa chuẩn hóa như sau: $Y=0,536+\mathbf{0 , 1 1 5} * K^{*} T^{*}+$ $\mathbf{0 , 4 2 9} * \mathbf{P L} * * *+\mathbf{0 , 1 7 6} * \mathrm{DD} * * *+\mathbf{0 , 1 5 0} * \mathbf{T N} * * *$.

Mức độ ảnh hưởng của 04 nhân tố CSR đến niềm tin của nhân viên các ngân hàng TMCP vào tổ chức tại thành phố Sóc Trăng - theo tầm quan trọng giảm dần: Trách nhiệm pháp luật (beta $=0,424)$, Trách nhiệm đạo đức (beta $=0,274)$, Trách nhiệm thiện nguyện (beta $=0,157)$, Trách nhiệm kinh tế (beta $=0,128)$.

Trong việc dò tìm sự vi phạm các giả định hồi quy tuyến tính: biểu đồ phân tán Scatterplot cho thấy phần dư không thay đổi theo một trật tự nào đối với giá trị dự đoán, chúng phân tán ngẫu nhiên, giả thuyết về liên hệ tuyến tính không bị vi phạm. Hệ số tương quan hạng Spearman của giá trị tuyệt đối phần dư và các biến độc lập: giá trị Sig. của các hệ số tương quan với độ tin cậy $95 \%$ đều lớn hơn 0,05 , cho thấy phương sai của sai số không thay đổi, giả định không bị vi phạm. Biểu đồ Histogram cho thấy phần dư có phân phối chuẩn với giá trị trung bình rất nhỏ gần bằng $0(\mathrm{Mean}=$ $9,66 \mathrm{E}-16)$ và độ lệch chuẩn của nó gần bằng $1(\mathrm{SD}=0,990)$, đồ thị $\mathrm{P}-\mathrm{P}$ plot biểu diễn các điểm quan sát thực tế tập trung khá sát đường chéo những giá trị kỳ vọng, có nghĩa là dữ liệu phần dư có phân phối chuẩn. Hệ số $1<$ Durbin - Watson $=1,765<3$ là thỏa điều kiện, các biến độc lập không có quan hệ chặt chẽ với nhau nên không xảy ra hiện tượng đa cộng tuyến. Như vậy, mô hình hồi quy tuyến tính được xây dựng theo phương trình trên không vi phạm các giả định hồi quy.

\section{KẾT LUẬN VÀ HÀM Ý QUẢN TRI}

\subsection{Kết luận}

Nghiên cứu sử dụng phương pháp chọn mẫu thuận tiện, 210 bảng khảo sát đạt yêu cầu phân tích và xử lý dữ liệu. Sau khi tiến hành kiểm định thang đo bằng công cụ Cronbach's Alpha và phân tích nhân tố khám phá EFA, mô hình nghiên cứu có 24 biến quan sát, trong đó thang đo nhận thức trách nhiệm xã hội có 20 biến gồm 4 thành phần: (1) nhận thức trách nhiệm kinh tế, (2) nhận thức trách nhiệm pháp luật, (3) nhận thức trách nhiệm đạo đức, (4) nhận thức trách nhiệm thiện nguyện; thang đo niềm tin tổ chức có 4 biến quan sát. Kết quả hồi qui cho thấy các yếu tố: (1) nhận thức trách nhiệm kinh tế, (2) nhận thức trách nhiệm pháp luật, (3) nhận thức trách nhiệm đạo đức, (4) nhận thức trách nhiệm thiện nguyện đều có tác động dương đến niềm tin tổ chức. Trong đó, yếu tố nhận thức trách nhiệm pháp luật có tác động mạnh nhất và yếu tố nhận thức trách nhiệm kinh tế có tác động thấp nhất.

\subsection{Hàm ý quản trị Về trách nhiệm pháp luật}


Đây là yếu tố tác động mạnh nhất đến niềm tin của nhân viên. Một số phương cách để thực hiện nội dung này: (1) Xây dựng quy chế khen thưởng trong hệ thống ngân hàng, (2) Xây dựng quy chế về công tác cán bộ trong hệ thống, (3) Ngân hàng nên lưu ý trong việc tạo ra cơ hội bình đẳng để mọi thành viên có thể phấn đấu, phát huy hết năng lực bản thân và gắn kết với nhau bằng tình cảm gắn bó, yêu thương, chia sẻ, (4) Xây dựng lộ trình phát triển nghề nghiệp cho từng vị trí công việc, (5) Ngân hàng nên áp dụng việc luân phiên chuyển các nhà quản lý trong hai hoặc ba năm nhằm để tránh sự thiên vị.

\section{Về trách nhiệm đạo đức}

Đây là yểu tố tác động mạnh thứ hai. Ngân hàng cần chú ý: (1) Các hoạt động của ngân hàng cần phải luôn tuân theo chuẩn mực đạo đức trong kinh doanh, (2) Ngân hàng cần luôn hợp tác với các khách hàng và đối tác theo quy tắc công bằng, đôi bên cùng có lợi, (3) Các ngân hàng cần xây dựng chuẩn mực đạo đức kinh doanh của mình và triển khai một cách rộng rãi, đồng bộ đến tất cả các bộ phận, các thành viên trong nội bộ biết và thực hiện, (4) Ngân hàng nên quan tâm đào tạo, huấn luyện thái độ tác phong, cũng như phong cách ứng xử của nhân viên, để phù hợp với văn hóa ngân hàng của mình.

\section{Về trách nhiệm thiện nguyện}

Đây là yếu tố tác động mạnh thứ 3 đến niềm tin của nhân viên vào tổ chức. Ngân hàng có thể quan tâm thực hiện một số biện pháp: (1) Xác định hoạt động từ thiện như là một phần tất yếu trong các hoạt động của ngân hàng, (2) Gắn kết với chính quyền địa phương và các cơ quan chức năng trong tổ chức các hoạt động từ thiện để đảm bảo đúng đối tượng và kịp thời, (3) Làm từ thiện ngay trong nội bộ ngân hàng, đối với những trường hợp nhân viên gặp hoạn nạn hay có hoàn cảnh khó khăn, (4) Thường xuyên tổ chức các hoạt động từ thiện để nhân viên có điều kiện tham gia.

\section{Về trách nhiệm kinh tế}

Đây là yểu tố tác động yếu nhất. Ngân hàng cần: (1) Xây dựng và hoạch định tầm nhìn, chiến lược dài hạn cho hoạt động của ngân hàng, (2) Xây dựng môi trường làm việc thoải mái, thân thiện, cởi mở, (3) Thực hiện chính sách động viên, khen thưởng và thi đua để khuyến khích tinh thần làm việc của nhân viên, (4) Chú trọng hơn công tác đào tạo, ngoài các khóa đào tạo các kỹ năng cơ bản thông qua chương trình đào tạo việc tại chỗ, cần bổ sung thêm các chương trình đào tạo và phát triển từ bên ngoài, (5) Quan tâm đến đời sống tinh thần của nhân viên, hỗ trợ những nhân viên có hoàn cảnh khó khăn.

\subsection{Hạn chế của đề tài}

Đề tài còn có những hạn chế nhất định: (1) Nghiên cứu sử dụng phương pháp chọn mẫu phi xác suất, lấy mẫu thuận tiện nên hạn chế tính tổng quát của đám đông, (2) Nghiên cứu chỉ đề cập đến tác động của trách nhiệm xã hội đến niềm tin của nhân viên trong tổ chức trong khi đó vẫn còn một vài kết quả khác chưa được xem xét đến như lòng trung thành, sự gắn kết..., (3) Phạm vi nghiên cứu là các ngân hàng TMCP tại TP. Sóc Trăng, chưa nghiên cứu ở những địa bàn đặc thù khác, hay phạm vi rộng hơn. Đó cũng chính là những gợi ý cho những nghiên cứu tiếp theo.

\section{TÀI LIỆ THAM KHẢO}

Bowen, R. (1953). Social responsibilities of the business man. New York: Harper.

Carroll, A. B. (1979). A three-dimensional conceptual model of corporate 
Performance. Academy of Management Review, 4(4), 497-505.

Carroll, A. B. (1991). The pyramid of corporate social responsibility: toward the moral management of organizational stakeholders. Business Horizons, 34, 39-48.

Cung, N. Đ., \& Đức, L. M. (2008). Trách nhiệm xã hội của Doanh nghiệp: Một số vấn đề lý luận và yêu cầu đổi mới trong quản lý nhà nước đối với trách nhiệm xã hội ở Việt Nam. Tạp chí Quản Lý Kinh tế, 23, 3- 11.

Bartels, F. L., Giao, H. N. K., \& Ohlenburg, T. J. (2006). ASEAN Multinational Entreprises: A Structural Analysis of Strategic Coherence. ASEAN Economic Bulletin, 23(2), 171-191. doi:10.1355/AE23-2C

Giao, H. N. K. (2010). Mạn đàm thước đo Văn hóa Doanh nghiệp trong các Ngân hàng thương mại. Tạp chí Ngân hàng, Ngân hàng Nhà nước VN, 22, 25-27. doi:10.31219/osf.io/rtqz4

Giao, H. N. K. (2012, 16/06/2012). Hoc chiến lược tùt doanh nghiệp siêu nhỏ- Tại sao không? Hỗ trợ Doanh nghiệp nhỏ và vừa vượt qua khủng hoảng, Trường Đại học Tôn Đức Thắng.

Giao, H. N. K., \& Lộc, N. Q. (2016). Ảnh hưởng của áp lực công việc và động lực làm việc đến hiệu quả công việc của nhân viên tại khách sạn Quê Hương Liberty. Tạp chí Du lịch Việt Nam, Tổng Cục Du lịch, 1-3. doi:10.31219/osf.io/szw37

Giao, H. N. K., \& Minh, H. V. (2016). Các yếu tố ảnh hưởng đến động lực làm việc của công nhân tại công ty Điện lực Tân Thuận. Tạp chí Công Thuơng-Bộ Công thuong, 10, 142-147. doi:10.31219/osf.io/z94v8

Giao, H. N. K., \& Mơ, N. T. H. (2016). Yêu cầu kỹ năng mềm của lao động trong thời kỳ hội nhập. Nhận diện cơ hội và thách thức đối với doanh nghiệp trong tiến trình hội nhập kinh tế quốc tế - Phân tích từ thị trường lao động, Bình Thuận.

Giao, H. N. K., \& Phong, N. L. D. (2015). Ảnh hưởng của công bằng trong tổ chức đến sự hài lòng đối với công việc của nhân viên tại trường Đại học Tài chính Marketing. Tạp chí Nghiên cứu Tài chính- Marketing, 29, 30-36. doi:10.31219/osf.io/mgd3r

Giao, H. N. K., \& Phương, V. T. M. (2011). Đo lường sự thỏa mãn công việc của nhân viên sản xuất tại công ty TNHH TM-DV Tân Hiệp Phát. Tạp chí Phát triển Kinh tế, 6, 15-21. doi:10.31219/osf.io/uwcae

Giao, H. N. K., \& Tài, L. T. T. (2016). Các nhân tố tác động đến gắn kết của nhân viên với tổ chức tại Trường Đại học Tài chính - Marketing. Tạp chí Nghiên cứu Tài chinh-Marketing, 34, 61-67. doi:10.31219/osf.io/9pm2v

Giao, H. N. K., \& Thu, V. T. K. (2017). Ảnh hưởng độ thỏa mãn công việc đến lòng trung thành của nhân viên các công ty tìm kiếm thăm dò khai thác dầu khí tại VN. Tạp chí Khoa học và Công nghệ- Đại học Công nghiệp TPHCM, 29(2), 130-147. doi:10.31219/osf.io/wdsgx

Giao, H. N. K., \& Trân, N. Đ. H. (2017). Các yếu tố ảnh hưởng đến sự gắn kết nhân viên với Trường Đại học Ngân hàng Thành phố Hồ Chí Minh. Tạp chí Công Thuong, 10, 246-251. doi:10.31219/osf.io/nswz6

Giao, H. N. K., \& Trang, H. T. T. (2016). Ảnh hưởng của văn hóa tổ chức đến sự gắn kết của nhân viên văn phòng tại tỉnh Bà Rịa - Vũng Tàu. Tạp chí Kinh tế-Kỹ thuật Bình Dưong, 14, 39-49. doi:10.31219/osf.io/mc3ka 
Giao, H. N. K., \& Vinh, N. H. (2015). Các yếu tố ảnh hưởng đến sự sáng tạo trong công việc của nhân viên Trường Đại học Tài chính - Marketing. Tạp chí Nghiên cúu Tài chính-Marketing, 31, 80-90. doi:10.31219/osf.io/kzavp

Giao, H. N. K., \& Vương, B. N. (2016). Ảnh hưởng của các yếu tố văn hóa doanh nghiệp đến sự gắn bó của nhân viên với tổ chức tại Công ty $\mathrm{CP}$ CMC Telecom TPHCM. Tạp chí Kinh tế- Kỹ thuật Bình Dưong, 13, 87-101. doi:10.31219/osf.io/qje82

Lee, Y., Kim, Y., Lee, K. H., \& Li, D. X. (2012). The impact of CSR on relationship quality and relationship outcomes: A perspective of service employees. International Journal of Hospitality Management, 31, 745- 756.

Mirza. M., \& Redzuan, M. (2012). The relationship between principal's leadership styles and teacher's organizational trust and commitment. Life Science Journal, 9(3), 1356-1362.

Quân, N. M. (2013). Lòng tin tổ chức- Một chỉ số quan trọng về năng lực doanh nghiệp: Khảo sát so sánh ở các doanh nghiệp quốc tế và doanh nghiệp Việt Nam. Tạp chí kinh tế và phát triển, 195, 18- 25.

Thảo, H. T. P., \& Hồ, H. L. (2015). Ảnh hưởng của nhận thức trách nhiệm xã hội đến niềm tin và cam kết gắn bó của nhân viên ngân hàng TMCP tại TP.HCM. Tạp chi phát triển kinh tế, 26(8), 37-53.

Trọng, H., \& Ngọc, C. N. M. (2008). Phân tích dữ liệu nghiên cứu với SPSS, Nhà Xuất Bản Hồng Đức. TPHCM.

Tschannen-Moran, M., Woolfolk, Hoy, A., \& Hoy, W. K. (1998). Teacher efficacy: Its meaning and measure. Review of Educational Research, 68, 202-248.

You, C. S, Huang, C. C, Wang, H. B, Liu, K. N, Lin, C. H., \& Tseng, J. S. (2013). The relationship between corporate social responsibility, job satisfaction and organizational commitment. The International Journal of Organizational Innovation, 5(4), 65-77. 